\section{Mutations in the CYP1B1 gene may contribute to juvenile-onset open-angle glaucoma}

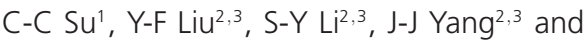
Y-C Yen ${ }^{4,5}$

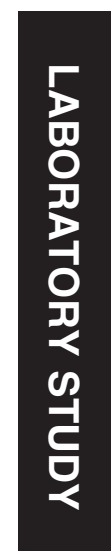

${ }^{1}$ Tian-Sheng Memorial Hospital, Tong Kang, Pin-Tong, Taiwan

${ }^{2}$ Department of BioMedical Sciences, Chung Shan Medical University, Taichung, Taiwan

${ }^{3}$ Department of Medical Research, Chung Shan Medical University Hospital, Taichung, Taiwan

${ }^{4}$ Department of Ophthalmology, Chi-Mei Medical Center, Liou-Ying, Tainan, Taiwan

${ }^{5}$ Department of Nursing, Min Hwei College of Health Care Management, Tainan, Taiwan

Correspondence: J-J Yang, Department of BioMedical Sciences, Chung Shan Medical University, No, 110, Sec.1 Chien-Kun N. Rd. Taichung 402, Taiwan

Tel: +886 424730022

Ext 11804;

Fax: +886 424757412

E-mail: jiannjou@csmu. edu.tw

or Y-C Yen, Department of Ophthalmology, Chi-Mei Medical Center, Liou-Ying,

Tainan, Taiwan

Tel:+886 6 6226999;

Fax: +886 62756011

E-mail: dy5101@yahoo. com.tw

Received: 2 November 2011 Accepted in revised form: 6 July 2012

Published online: 10 August 2012 
optineurin and the WDR36 gene encoding a protein of unknown function, seem to harbour the mutations that lead to POAG. ${ }^{5-7}$ Additionally, CYP1B1 has been identified as a causative gene in primary congenital glaucoma (PCG) for nearly a decade. ${ }^{7}$ Recent evidence has suggested the involvement of CYP1B1 mutations in several forms of glaucoma and anterior segment disorders. ${ }^{8}$ On the basis of the above observations, CYP1B1 seems to have a wide role in ocular physiology.

CYP1B1 belongs to the CYP450 superfamily that contains 58 and 102 putatively functional genes in the human and mouse genome, respectively. Human CYP1B1 was the first gene in the CYP450 gene superfamily in which a mutation was demonstrated to be involved in a primary developmental defect. ${ }^{9}$ Although it was mapped to chromosome 2p22-p21 by fluorescence in situ hybridization, the CYP1B1 gene contains three exons and two introns. Initiated in exon 2, the putative open reading frame was $1629 \mathrm{bp} .{ }^{10}$ The CYP1B1 gene encoded a 543-amino-acid-long protein, including a membrane-bound region consisting of 53 residues in N-terminal, a 10-residue-long proline-rich region called a hinge, and a cytosolic globular domain consisting of 480 amino acids. ${ }^{11}$ The CYP1B1 gene is expressed in several tissues, including the eye, as well as in the nucleus of several cell types, including tubule cells of the kidney and secretory cells of the breast. ${ }^{12}$ In a recent study, mutations in MYOC and CYP1B1 genes were implicated in POAG and PCG, respectively. ${ }^{13}$ Another study indicated that a digenic inheritance of CYP1B1 and MYOC mutations results in a phenotype with more pronounced glaucoma, suggesting that CYP1B1 may function as a gene modifier for the MYOC gene. ${ }^{14}$ However, other studies indicated that CYP1B1 gene alone could be responsible for JOAG, in French, ${ }^{15}$ Indian, ${ }^{16}$ and Spanish ${ }^{17}$ patients. A homozygous p.G61E missense mutation of CYP1B1 was also reported in the familial juvenile glaucoma. ${ }^{18}$ The above studies demonstrate that CYP1B1 mutations potentially threaten JOAG and might also modify the glaucoma phenotype in patients who do not carry a MYOC mutation. Our previous study found that mutations of $M Y O C$ and OPTN are responsible for $\sim 12.5 \%$ of JOAG in the Taiwan population. ${ }^{19,20}$ However, exactly what causes the other $87.5 \%$ remains unclear. Further studies are warranted to determine whether other genes have mutations that account for a significant proportion of JOAG in the Taiwan population. Therefore, this study investigated the $5^{\prime}-, 3^{\prime}$-untranslated (UTR) and coding regions of the CYP1B1 gene in 61 JOAG patients and 100 normal unrelated individuals, and tried to determine whether mutations in that gene are associated with the development of JOAG.

\section{Materials and methods}

\section{Subject selection}

A total of 61 individuals with JOAG were subjected to analysis of mutations in this study. All study patients received more than two complete ocular examinations, each comprising slit-lamp testing, IOP measurement, fundus examination and visual-field examination. Patients were defined as suffering from JOAG, if they were first diagnosed younger than 35-years-old and found to have an IOP $>22 \mathrm{~mm} \mathrm{Hg}$, a cup/disc ratio $>0.5$ or optic nerve asymmetric, a visual-field loss characteristic of glaucomatous change, and an open angle width ranging from Shaffer grade II to IV without any other apparent secondary cause (eg, traumatically or surgically induced). Most of these subjects have been reported in a previous study of MYOC and OPTN mutations in Taiwanese patients with JOAG. ${ }^{19,20}$ Subjects with mutations in the MYOC gene were also included in the study.

One hundred, randomly selected, normal individuals over 50 years of age were included as the control group from the Department of Aged Physical Checkup in the Chi Mei Medical Centre. Individuals in the control group also received complete ocular examinations, as described above, to exclude the possibility of glaucoma and other opthalmology disease. All of 161 individuals in the study, 61 patients and 100 controls, belong to the Han ethnic origin. The study protocol was approved by the Institutional Review Board (IRB) of Chi Mei Medical Centre and was carried out in accordance with the World Medical Association's Declaration of Helsinki (2000). All patients provided signed, informed consent to study participation, subsequent to the details of the study having been explained in detail to them.

\section{Detection of the mutations of the CYP1B1 gene}

DNA samples were collected from $10 \mathrm{ml}$ of peripheral blood acquired from each of the 161 individuals and purified using a Gentra DNA Blood Kit (Gentra Systems, Inc., Minneapolis, MN, USA), according to the manufacturer's directions. The quality and quantity of purified genomic DNA were determined by gel electrophoresis and spectrophotometry, respectively. Mutations in the $5^{\prime}-,-33^{\prime}$-UTR and coding regions of the CYP1B1 gene were screened by direct sequencing (GenBank Accession No.NM_000104). Intragenic primers used for polymerase chain reaction (PCR) are listed in Table 1. In brief, PCR was carried out in a reaction volume of $25 \mu \mathrm{l}$ containing $100 \mathrm{ng}$ of genomic DNA, $200 \mu \mathrm{M}$ dNTP, 0.25 units of proTaq DNA polymerase (Promega Corporation, Madison, WI, USA), and $200 \mu \mathrm{M}$ intragenic primers. The PCR products were purified 
Table 1 Oligonucleotide Primer Pairs for PCR

\begin{tabular}{llccc}
\hline Name & Oligonucleotide & Annealing temperature $\left({ }^{\circ} \mathrm{C}\right)$ & Region & Product size $($ bp $)$ \\
\hline E1F & 5'-GTCAAAGCGGCCTGGTGT-3' & 64 & Exon 1 & 632 \\
E1R & 5'-TAACGGTTCCTGCAATCTGG-3' & & & Exon 2 \\
E2F & 5'-TCTCCAGAGAGTCAGCTCCG-3' & 63 & & 1230 \\
E2R & 5'-CTACTCCGCCTTTTTCAGA-3' & 60.2 & Exon 3 & 844 \\
E3F & 5'-GTCACTGAGCTAGATAGCCT-3' & & & \\
E3R & 5'-GGACAGTTGATTTATGCTCACC-3' & & \\
\hline
\end{tabular}

using a PCR Purification Kit (QIAGEN GmbH, Hilden, Germany) and then subjected to PCR-directed DNA sequencing using a DNA Sequencing Kit (Applied Biosystems Corporation, Foster City, CA, USA).

Sequencing was performed on an Applied Biosystems model 3730 automated sequencer (Applied Biosystems Corporation). Sequence data were compared with the published sequence of the CYP1B1 gene (GenBank Accession No.NM_000104).

\section{Statistical analysis}

Significant differences in allele frequencies between JOAG patients and controls were determined by the $\chi^{2}$ test. A $P$-value less than 0.05 represented a statistically significant difference between JOAG patients and controls.

\section{Model building and structural-based analysis}

Human CYP1B1 gene, which has been elucidated by X-ray crystallography (PDB entry 3PM0) to a resolution of $2.7 \AA$, provides a suitable template. ${ }^{21}$ Threedimensional (3D) modelling of the human wild-type and p.R390H mutation were performed using SWISSMODEL, an automated homology modelling program. The homology-modelling server SWISS-MODEL is directly accessible at http:/ / swissmodel.expasy.org/ workspace/. ${ }^{22,23}$ This study used the automatic modelling approach to apply the complete protein sequence of human CYP1B1, including its 543 amino acids and its mutation, which are available in NCBI GenBank (NP_000095.2) in FASTA format. Data obtained by the homology models were visualized using Accelrys Viewer Lite version 5.0 (Accelrys, Inc., San Diego, CA, USA).

\section{Results}

Mutations in three exons of the CYP1B1 gene (GenBank Accession No.NM_000104), including flanking intronic sequences, 5'-UTR, 3'- UTR region and coding region, were screened by PCR amplification and direct DNA sequence analysis. Five polymorphic sites of the CYP1B1 gene were identified in the 61 JOAG patients and 100 unrelated normal controls (Table 2). We made statistical $\chi^{2}$ test, comparison of the allelic frequencies on these polymorphic sites found in the JOAG patients and the controls. Allele frequencies of most polymorphisms between the patients and the controls were not different significantly $(P>0.05)$, except for variant c.403-13C $>\mathrm{T}$ $(P=0.04<0.05$; Table 2$)$.

Although none were found in the normal controls, two mutations of the CYP1B1 gene were identified in 3 of the 61 JOAG patients (Figure 1). The prevalence of CYP1B1 gene mutations in this study was $4.92 \%(3 / 61)$. A heterozygous c.1-313A $>\mathrm{C}$ mutation was found in one patient $(1 / 61 ; 1.64 \%$; Figure $1 \mathrm{~b})$, and two patients had homozygous c.1169G > A mutation $(2 / 61 ; 3.28 \%$; Figure 1d). Among the two mutations, the c.1169G $>$ A mutations located in the coding region led to amino-acid changes within the CYP1B1 protein. c.1169G $>$ A is a transversion mutation that leads to arginine (Arg; R) $\rightarrow$ histidine (His; H) substitution at codon 390 (p.R390H). Additionally, a heterozygous c.1-313A > C mutation was found in the $5^{\prime}$-UTR of CYP1B1 gene (Figure 1e).

To understand the role and effect of the p.R390H missense mutation, we examined amino-acid sequences of CYP1B1 using a basic ConSeq analysis system (http:/ / conseq.tau.ac.il/). Following deposition of the protein sequence of CYP1B1 into the system, the system automatically detected homologous sequences of CYP1B1 and conducted multiple alignments. In total, 503 PSI-BLAST hits were detected by the system, of which 496 were unique sequences. Next step, the system automatically calculated the 50 sequences with the lowest E-values. Analytical results indicated that p.R390 residue is exposed and highly conserved (Conseq score $=9$ ) in the CYP1B1 protein of all species (Figure 2).

To further study the protein-level mechanisms of the p.R390H mutation, a 3D model was constructed for a bioinformatic structural analysis. Analytical results revealed that the parallel orientation of side chains of Arg390 and Glu387 located at the helical K domain of CYP1B1, which are formed a hydrogen-bonding interaction with the turn-apart meander region end of Asn428 (Figure 3a). This is expected to be involved in the 
Table 2 The polymorphisms of CYP1B1 gene in Study

\begin{tabular}{|c|c|c|c|c|c|c|}
\hline \multirow{2}{*}{$\begin{array}{l}\text { Variants } \\
\text { Genotype }\end{array}$} & \multicolumn{2}{|c|}{ Allele frequency (\%) } & \multicolumn{2}{|c|}{ Genotype frequency } & \multirow[t]{2}{*}{$\chi^{2}(d f=1)$} & \multirow[t]{2}{*}{$\mathrm{P}^{*}$} \\
\hline & $J O A G(n=122)$ & Control $(n=200)$ & $J O A G(n=61)$ & Control $(n=100)$ & & \\
\hline c. $403-13 \mathrm{C}>\mathrm{T}$ & C:0.902T:0.098 & C:0.825T:0.175 & $0 / 12 / 49$ & $0 / 35 / 65$ & 4.31 & 0.04 \\
\hline c. $142 \mathrm{C}>\mathrm{G}$ & C:0.869G:0.131 & C:0.830G:0.170 & $0 / 16 / 45$ & $0 / 34 / 66$ & 1.07 & 0.30 \\
\hline c. $355 \mathrm{G}>\mathrm{T}$ & G:0.861T:0.139 & G:0.840T:0.160 & $0 / 17 / 44$ & $0 / 32 / 68$ & 0.31 & 0.58 \\
\hline c. $1294 G>C$ & G:0.041 C:0.959 & G:0.100 C:0.900 & $56 / 5 / 0$ & $82 / 16 / 2$ & 3.41 & 0.18 \\
\hline c. $1347 \mathrm{~T}>\mathrm{C}$ & T:0.041 C:0.959 & T:0.095 C:0.905 & $56 / 5 / 0$ & $83 / 15 / 2$ & 2.97 & 0.23 \\
\hline
\end{tabular}

${ }^{*} \chi^{2}$ test was used.

${ }^{\&}$ The numbers indicated homozygote/heterozygote/wild type.
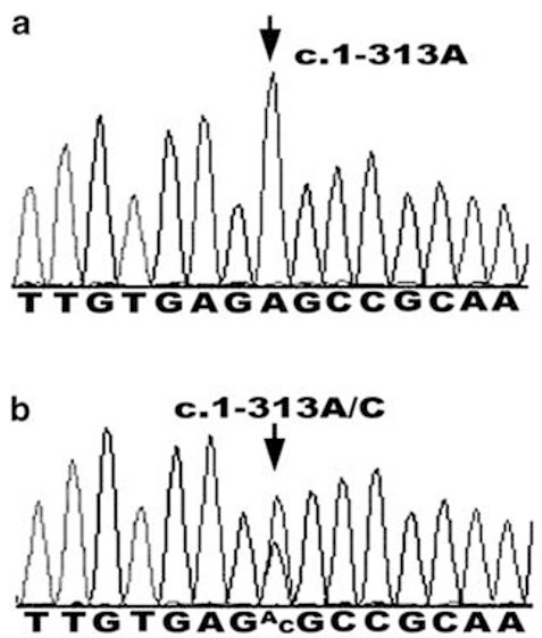

C

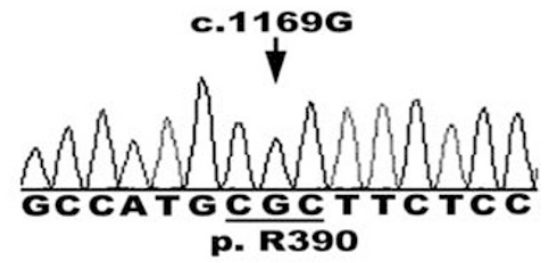

d

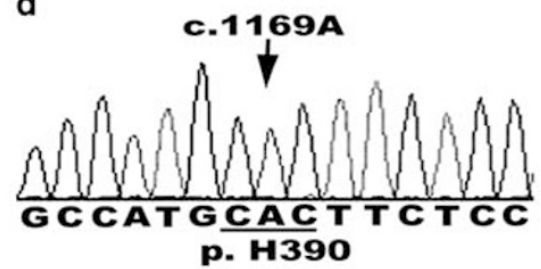

$\underline{\mathrm{e}}$

\begin{tabular}{ccccc}
\hline Genotype & Codon & Individual found & Percentage (\%) & Allele frequency \\
\hline c.1-313A $>$ C & $5{ }^{\prime}-$ UTR & 1 & $1.64 \%$ & A:0.992 C: 0.008 \\
c.1169G $>$ A & p.Arg390His & 2 & $3.28 \%$ & G:0.967 A:0.033 \\
\hline Total & & 3 & $4.92 \%$ & \\
\hline
\end{tabular}

Figure 1 Mutation analysis of CYP1B1 in 61 JOAG patients. Genomic sequence of the CYP1B1 from normal individuals (a and c); a variant on the promoter region at position - 313, heterozygous of c.1-303A $>$ C (b). A homozygous missense variant of c.1169G $>$ A (d). Vertical arrows denote the changes of the nucleotide. Underlining represents the amino-acid codon. (e) Prevalence of CYP1B1 mutations in the 61 JOAG individuals.

haem-binding and proper folding of the molecule. While histindine $(\mathrm{H})$ is to substitute arginine $(\mathrm{R})$ at codon 390, we found that the parallel orientation of the Glu387 and His390 residues side-chain-interaction dramatically change to the end of Asn428. In contrast, Trp404 and His390 side-chain interaction was more transparent in the 3D molecular models (Figure 3b). Therefore, we suggest that the p.R390H mutation may destroy the intramolecular structure of the CYP1B1 protein.

Previously studies show that both PolyPhen (Polymorphism Phenotyping) program and SIFT (Sorting tolerant from intolerant) algorithm methods can predict whether a single amino-acid substitution affects protein function. ${ }^{24,25}$ To assess the effect of p.R390H substitution, PolyPhen program and SIFT algorithm were also used in this study. Analytical results revealed that the PSIC (position-specific independent counts) score of p.R390H was 2.799 by PolyPhen program derived from multiple sequence alignment of observation. The PSCI score $>2.0$ was predicted that p.R390H mutation is damaging, which is supposed to affect protein function and structure. In addition, the SIFT score of p.R390H was 0.0001. The score less than 0.05 was predicted to affect protein function. Therefore, we suggest that substitution 


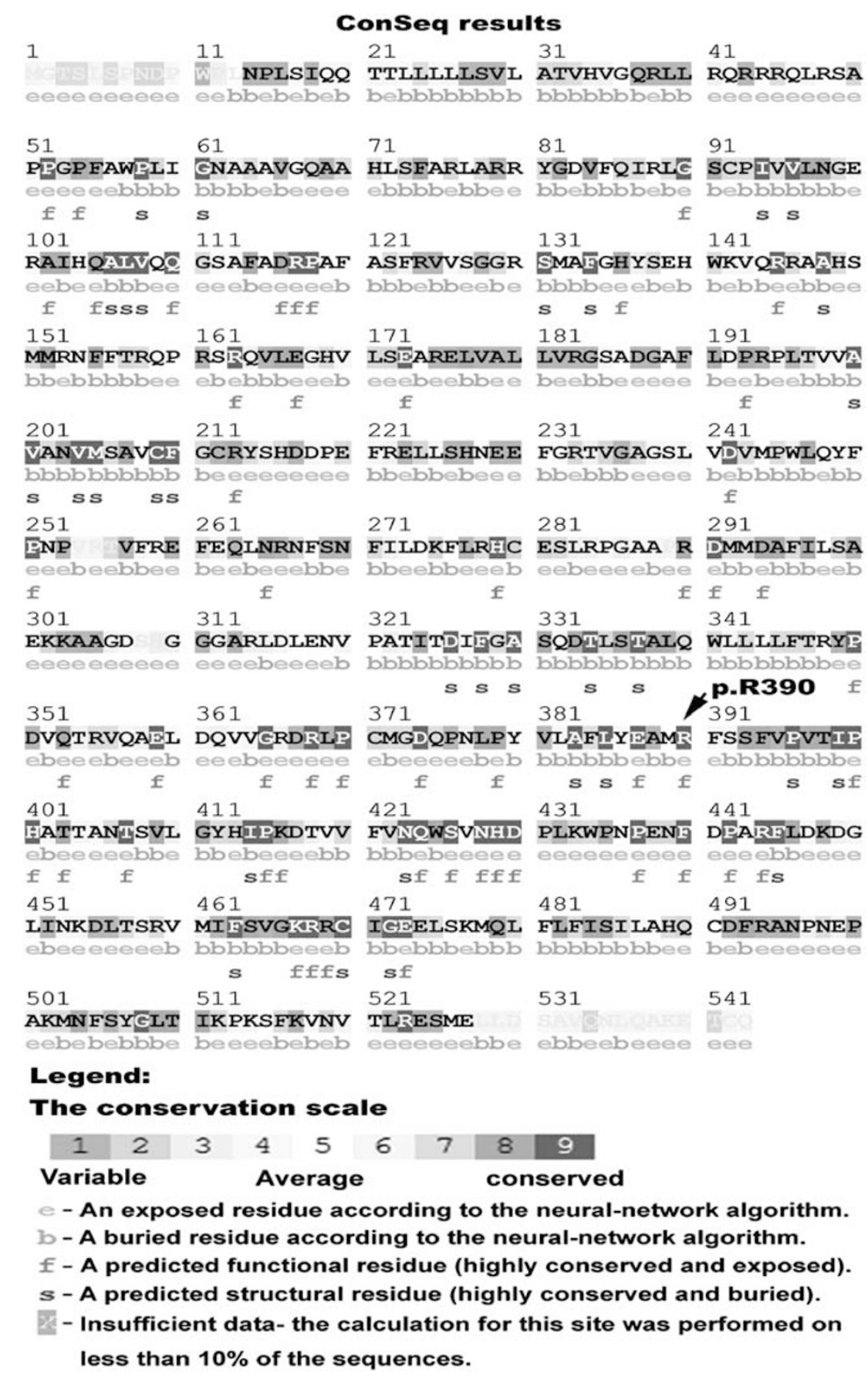

Figure 2 ConSeq predictions observed on human CYP1B1 (SWISS-PROT:Q16678 (CP1B1_Human)), using 50 homologues obtained from the Pfam database (family code: PF00067). The CYP1B1 protein sequence is displayed with the evolutionary rates at each site colour-coded onto it (see legend). The residues of the CYP1B1 sequence are numbered, starting from 1 . The first row below the sequence lists the estimated burial status of the site (ie, ' $b$ ', buried versus ' $e$ ', exposed). The second row denotes the residues predicted to be structurally and functionally important: 's' and 'f', respectively. Vertical arrows represent amino-acid codons (p.R390).

at codon 390 from $\mathrm{R}$ to $\mathrm{H}$ is predicted to be deleterious for the protein function by SIFT server. On the basis of above results, we postulate that p.R390 has an important role in CYP1B1 protein. In particular, mutant p.R390H incurs a loss of function in the CYP1B1 protein.
This study also attempted to determine whether the c.1-313 A > C promoter variant affects the transcription level by predicting the transcription factor binding sites of CYP1B1 extended within 50 bps of up and down of the c.1-313 A > C promoter region, including the variant site, 


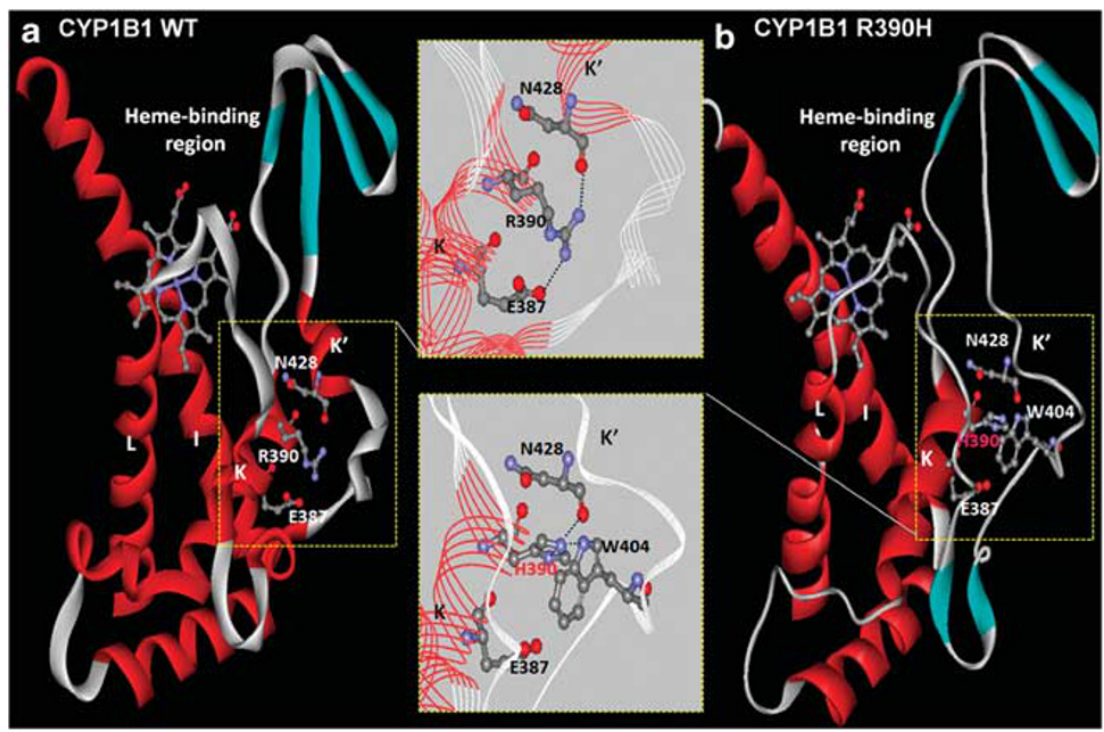

Figure 3 Bioinformatic estimation of the 3D structure of p.R309H CYP1B1 protein using human wild-type CYP1B1 protein (PDB ID 3PM0) as a template. Comparing the protein secondary structure architectures reveals the intra-molecular interactions between wildtype (a) and p.R390H mutation (b) in monomeric CYP1B1. Secondary structures are drawn with red solid ribbons ( $\alpha$-helices), blue ribbons ( $\beta$-sheets) and white loops (turns). The yellow boxes present the close-up views of the 'meander' region defined by a previous study focus on the C-terminal of theme-binding domain in a similar orientation and key residues as in ball-and-stick representation. Hydrogen bonding is denoted by dash lines. The highly conserved residues region, including Glu387, Arg390, and Asn428 located on helical turn apart, which was contributed from a hydrogen-bonding interaction to stabilize the 'meander' region structure, is essential for normal functioning of the P450 molecule.

based on use of the TFSEARCH prediction system (http:/ / www.cbrc.jp/research/db/TFSEARCH.html). ${ }^{26}$ No known transcription binding sites within this region were identified (data not shown).

\section{Discussion}

Nearly 100 mutations of CYP1B1 coding sequence have been found in patients with infantile congenital glaucoma among populations worldwide, including missense, nonsense, deletion, insertion or duplication mutations. ${ }^{11,27-30}$ According to previous study, CYP1B1 significantly contributes to PCG, a rare and severely blinding disease with recessive inheritance. ${ }^{11}$ Recent studies also indicated that the CYP1B1 gene alone could be responsible for POAG or JOAG patients. ${ }^{15-17}$ In this study, five variants of the CYP1B1 gene were found in the 61 JOAG patients and in the 100 unrelated normal controls. These sequence changes are not classified as causative mutations, but as polymorphisms, because the frequencies were similar between the patient and normal control groups. Therefore, these polymorphic sites alone did not provide any significant evidence of association with JOAG risk. However, we cannot rule out the possibility that an unrecognized difference in ancestry may exist between the case and control groups, or that the variants are in linkage disequilibrium with a true disease-causing variant nearby. In addition, 3 of the 61 $(3 / 61,4.92 \%)$ JOAG patients carried mutations of the CYP1B1 gene, including c.1-313A $>$ C and c.1169G $>A$ (p.R390H) mutation. Despite having a low frequency, those mutations were undetected in the 100 normal controls. Although previous studies have described CYP1B1 mutation as a modifier of the MYOC gene in the development of the glaucomatous process, 31,32 we did not find that the mutations of both MYOC and CYP1B1 were simultaneously presented in the same JOAG patient from this study and our previous study. ${ }^{19}$

Previously, p.R390H missense mutation has been described in one PCG case. ${ }^{33}$ Located in the conserved alpha helix K domain, the arginine (R) 390 residue forms the consensus sequence GluXXArg, which is conserved among all members of the cytochrome P450 superfamily. ${ }^{33}$ Similarly, our results indicated that p.R390 residue is highly conserved in the alpha helix $\mathrm{K}$ domain using the ConSeq server. ConSeq, a website server, can identify biologically important residues in a protein sequence. ${ }^{34}$ On the basis of this finding, the transversion of Arginine to Histidine at codon 390 of the CYP1B1 profoundly impacts the function of the CYP1B1 protein.

The 3D characterization of protein structures must be improved to elucidate the functions of proteins and their disease-formation relationships. ${ }^{22,35}$ High-resolution characterization of proteins can be provided by either 
experimental methods such as X-ray crystallography and nuclear magnetic resonance or by computational analysis. ${ }^{35}$ According to a previous prediction, the presence of p.R390 at conservation of this motif position is essential for the normal function of the CYPB1 molecule. ${ }^{33}$ In addition to altering the side-chain characteristics from linear guanidinium group to imidazole ring, the point mutation of p.R390H affects the formation of salt bridges located on the 'meander' region, which is essential for normal functioning of the CYP1B1 molecule. ${ }^{33}$ A recent study determined human CYP1B1 based on X-ray crystallography to $2.7 \AA$ resolution with $\alpha$-naphthoflavone bound in the active site cavity. $^{21}$ The structure of human CYP1B1 by X-ray diffraction can provide further insight into functional similarities and differences exhibited by family I P450s. ${ }^{21}$

Without the p.R390H missense mutation crystallographic structure, this study developed a mutation molecular 3D model structure of human CYP1B1 protein by using a simulated model with human CYP1B1 protein (PDB ID is 3PM0) as a template and an automatic SWISSMODEL homology modelling program. ${ }^{23}$ As the most accurate computational method to generate reliable structural models, homology modelling is conventionally used in many biological applications. ${ }^{20}$ Homology models of a protein of interest are a valuable means of interpreting sequence variations and designing mutagenesis experiments to elucidate the biological functions of proteins. ${ }^{36,37}$ This study has established that these highly conserved residues in alpha-helix $\mathrm{K}$ domain region including Glu387, Arg390, and Asn428 located on helical turn apart, which was created from a hydrogenbonding interaction to stabilize the 'meander' region structure, are essential for the normal function of the CYP1B1 molecule. The above results are similar and further confirm the previous prediction results. ${ }^{33}$ In addition, we found that p.R390 is important positions in the CYP1B1 protein sequence using PolyPhen program and SIFT algorithm prediction methods. p.R390 residue was conserved throughout evolution and, therefore, substitutions at these positions may affect protein function. Our clinical and gene analysis data reveal the mutation of this residue, p.R390H, in two patients with JOAG. Therefore, our prediction results obtained by homology modelling, PolyPhen program and SIFT algorithm prediction methods can elucidate the functional deficiencies of CYP1B1 to cause glaucoma.

Thirteen variants of $5^{\prime}$-UTR in the CYP1B1 gene have been described so far (http://www.ensembl.org/ Homo_sapiens/Gene/Variation_Gene/). Conversely, this study did not detect any of the above-mentioned variants. Instead, a novel variant, c.1-313A > C, was identified in one of our patients. We believe that differences between the variants of CYP1B1 are possibly owing to racial variation. The c.1-313A $>\mathrm{C}$ variant is located within the promoter region. A variant of promoter generally influences (upregulated or downregulated) the initiation of transcription. The influence of this variant at the transcript cannot be eliminated, despite the lack of known transcription binding sites within $50 \mathrm{bp}$ of up and down the c.1-313A site in our prediction assay by bioinformatics. Additionally, the variants of CYP1B1 were undetected in the 100 normal controls. Therefore, we posit that c.1$313 \mathrm{~A}>\mathrm{C}$ variant may be associated with JOAG. However, exactly how this variant affects the transcription and the promoter activity must be further understood, to more thoroughly understand the pathogenic role of CYP1B1 c.1-313A > C variant in JOAG.

Finally, we have provided comprehensive genetic information on the CYP1B1 from study subjects with JOAG in Taiwan. Our results indicate that mutations of CYP1B1 gene are responsible for about $4.92 \%(3 / 61)$ of JOAG in the population of Taiwan. Therefore, CYP1B1 gene likely is not a major genetic factor underlying JOAG. In addition, our previous results found that mutations in $M Y O C$ and OPTN gene of JOAG patients are $\sim 12.5 \%$ in the Taiwan population. ${ }^{19,20}$ Therefore, about $17.42 \%$ of JOAG patients were found at least a risk factor for JOAG development in the population of Taiwan. However, the cause of the remaining $82.58 \%$ remains elusive. Further studies are necessary to determine whether there are other gene mutations or evironmental risk factors for JOAG in the population of Taiwan.

\section{Summary}

What was known before

- Glaucoma is one of the leading causes of blindness in the world. Juvenile-onset open-angle (JOAG) is a subtype of glaucoma. In recent studies, mutations in $M Y O C$ and CYP1B1 genes were implicated in POAG, PCG and JOAG, respectively. However, little is known about the contribution of variants in the CYP1B1 gene to JOAG in Taiwan.

What this study adds

- Investigation of mutations in the CYP1B1 amongst patients suffering JOAG of Taiwan.

- Analysis results indicated two mutations, p.Arg390His and c.1-313A > C), in the JOAG patients.

- Computer-assisted modelling suggested that p.Arg390His affect protein structure and function.

- p.Arg390His mutation in CYP1B1 may be a risk factor for the development of JOAG in Taiwanese.

\section{Conflict of interest}

The authors declare no conflict of interest. 


\section{Acknowledgements}

We would like to thank all of the subjects who participated in the present project. This work is supported by Chi-Mei Medical Centre Liouying Research Grant (CLFHR 9821) and Chung Shan Medical University, Tian-Sheng Memorial Hospital (CSMUTSMH- 099-001). Ted Knoy is appreciated for his editorial assistance.

\section{References}

1 Stone EM, Fingert JH, Alward WL, Nguyen TD, Polansky JR, Sunden SL et al. Identification of a gene that causes primary open angle glaucoma. Science 1997; 275: 668-670.

2 Fuse N. Genetic bases for glaucoma. Tohoku J Exp Med 2010; 221: 1-10.

3 Turalba AV, Chen TC. Clinical and genetic characteristics of primary juvenile-onset open-angle glaucoma. Semin Ophthalmol 2008; 23: 19-25.

4 Morissette J, Cote G, Anctil JL, Plante M, Amyot M, Heon E et al. A common gene for juvenile and adult-onset primary open-angle glaucomas confined on chromosome 1q. Am J Hum Genet 1995; 56(6): 1431-1442.

5 Sheffield VC, Stone EM, Alward WL, Drack AV, Johnson AT, Streb LM et al. Genetic linkage of familial open angle glaucoma to chromosome 1q21-q31. Nat Genet 1993; 4 47-50.

6 Stoilova D, Child A, Brice G, Crick RP, Fleck BW, Sarfarazi M. Identification of a new 'TIGR' mutation in a family with juvenile-onset primary open angle glaucoma. Ophthalmic Genet 1997; 18: 109-118.

7 Gong G, Kosoko-Lasaki O, Haynatzki GR, Wilson MR. Genetic dissection of myocilin glaucoma. Hum Mol Gnent 2004; 13: R91-R102.

8 Choudhary D, Jansson I, Schenkman JB. CYP1B1, a developmental gene with a potential role in glaucoma therapy. Xenobiotica 2009; 39(8): 606-615.

9 Nelson DR, Zeldin DC, Hoffman SM, Maltais LJ, Wain HM, Nebert DW. Comparison of cytochrome P450 (CYP) genes from the mouse and human genomes, including nomenclature recommendations for genes, pseudogenes and alternative-splice variants. Pharmacogenetics 2004; 14: 1-18.

10 Tang YM, Wo YYP, Stewart J, Hawkins AL, Griffin CA, Sutter TR et al. Isolation and characterization of the human cytochrome P450 CYP1B1 gene. J Biol Chem 1996; 271: 28324-28330.

11 Vasiliou V, Gonzalez FJ. Role of CYP1B1 in Glaucoma. Annu Rev Pharmacol Toxicol 2008; 48: 333-358.

12 Muskhelishvili L, Thompson PA, Kusewitt DF, Wang C, Kadlubar FF. In situ hybridization and immunohistochemical analysis of cytochrome P450 1B1 expression in human normal tissues. J Histochem Cytochem 2001; 49: 229-236.

13 Acharya M, Mukhopadhyay A, Bhattacharjee A, Thakur SK, Bandyopadhyay AK, Ray K. Complex genetics of glaucoma: defects in CYP1B1, and not MYOC, cause pathogenesis in an early-onset POAG patient with double variants at both loci. J Genet 2008; 87(3): 265-269.

14 Vincent AL, Billingsley G, Buys Y, Levin AV, Priston M, Trope $\mathrm{G}$ et al. Digenic inheritance of early-onset glaucoma:
CYP1B1, a potential modifier gene. Am J Hum Genet 2002; 70: 448-460.

15 Melki R, Colomb E, Lefort N, Brezin AP, Garchon HJ. CYP1B1 mutations in French patients with early-onset primary open-angle glaucoma. J Med Genet 2004; 41: 647-651.

16 Acharya M, Mookherjee S, Bhattacharjee A, Bandyopadhyay AK, Thakur SKD, Bhaduri G et al. Primary role of CYP1B1 in Indian juvenile-onset POAG patients. Mol Vis 2006; 12: 399-404.

17 López-Garrido MP, Sánchez-Sánchez F, López-Martínez F, Aroca-Aguilar JD, Blanco-Marchite C, Coca-Prados M et al. Heterozygous CYP1B1 gene mutations in Spanish patients with primary open-angle glaucoma. Mol Vis 2006; 12: 748-755.

18 Khan AO, Lama Al-Abdi, Mohamed JY, Aldahmesh MA, Alkuraya FS. Familial juvenile glaucoma with underlying homozygoud p.G61E CYP1B1 mutations. J AAPOS 2011; 15: 198-199.

19 Yen YC, Yang JJ, Chou MC, Li SY. Identification of mutations in the myocilin (MYOC) gene in Taiwanese patients with juvenile-onset open-angle glaucoma. Mol Vis 2007; 13: 1627-1634.

20 Yen YC, Yang JJ, Chou MC, Li SY. Absence of OPTINEURIN $(O P T N)$ gene mutations in Taiwanese patients with Juvenile-Onset Open-Angle Glaucoma. Mol Vis 2008; 14: 487-494.

21 Wang A, Savas U, David Stout C, Johnson EF. Structural Characterization of the Complex between $\alpha$-Naphthoflavone and Human Cytochrome P450 1B1. J Bio Chem 2011; 286: 5736-5743.

22 Arnold K, Bordoli L, Kopp J, Schwede T. The SWISSMODEL workspace: a web-based environment for protein structure homology modelling. Bioinformatics 2006; 22: 195-201.

23 Bordoli L, Kiefer F, Arnold K, Benkert P, Battey J, Schwede T. Protein structure homology modeling using SWISS-MODEL workspace. Nat Protoc 2009; 4: 1-13.

24 Ramensky V, Bork P, Sunyaev S. Human non-synonymous SNPs: server and survey. Nucleic Acids Res 2002; 30: 3894-3900.

25 Kumar P, Henikoff S, Ng PC. Predicting the effects of coding non-synonymous variants on protein function using the SIFT algorithm. Nat Protoc 2009; 4: 1073-1081.

26 Heinemeyer T, Wingender E, Reuter I, Hermjakob H, Kel AE, Kel OV et al. Databases on Transcriptional Regulation: TRANSFAC, TRRD, and COMPEL. Nucleic Acids Res 1998; 26: $364-370$

27 Firasat S, Riazuddin SA, Khan SN, Riazuddin S. Novel CYP1B1 mutations in consanguineous Pakistani families with primary congenital glaucoma. Mol Vis 2008; 14: 2002-2009.

28 Yang M, Guo X, Liu X, Shen H, Jia X, Xiao X et al. Investigation of CYP1B1 mutations in Chinese patients with primary congenital glaucoma. Mol Vis 2009; 15: 432-437.

29 Weisschuh N, Wolf C, Wissinger B, Gramer E. A clinical and molecular genetic study of German patients with primary congenital glaucoma. Am J Ophthalmol 2009; 147: 744-753.

30 Campos-Mollo E, López-Garrido MP, Blanco-Marchite C, Garcia-Feijoo J, Peralta J, Belmonte-Martínez J et al. CYP1B1 mutations in Spanish patients with primary congenital 
glaucoma: phenotypic and functional variability. Mol Vis 2009; 15: 417-431.

31 Bejjani BA, Stockton DW, Lewis RA, Tomey KF, Dueker DK, Jabak $\mathrm{M}$ et al. Multiple CYP1B1 mutations and incomplete penetrance in an inbred population segregating primary congenital glaucoma suggest frequent de novo events and a dominant modifier locus. Hum Mol Genet 2000; 9: 367-374.

32 Vincent AL, Billingsley G, Buys Y, Levin AV, Priston M, Trope $\mathrm{G}$ et al. Digenic inheritance of early-onset glaucoma: CYP1B1, a potential modifier gene. Am J Hum Genet 2002; 70: $448-460$

33 Stoilov I, Akarsu AN, Alozie I, Child A, Barsoum-Homsy M, Turacli ME et al. Sequence analysis and homology modeling suggest that primary congenital glaucoma on 2 p21 results from mutations disrupting either the hinge region or the conserved core structures of cytochrome P4501B1. Am J Hum Genet 1998; 62: 573-584.

34 Berezin C, Glaser F, Rosenberg J, Paz I, Pupko T, Fariselli P et al. ConSeq: the identification of functionally and structurally important residues in protein sequences. Bioinformatics 2004; 20: 1322-1324.

35 Sasin JM. Bujnicki JM. COLORADO3D, a web server for the visual analysis of protein structures. Nucleic Acids Res 2004; 32: W586-W589.

36 Peitsch MC. About the use of protein models. Bioinformatics 2002; 18: 934-938.

37 Junne T, Schwede T, Goder V, Spiess M. The plug domain of yeast Sec61p is important for efficient protein translocation, but is not essential for cell viability. Mol Biol Cell 2006; 17: $4063-4068$. 